\title{
Optimal Routing Strategy of Wireless Sensor Network for Prolonging Lifetime
}

\author{
Mei Wang, Wen-Yuan Chen, ${ }^{1 *}$ and Liang Huang \\ College of Electrical and Control Engineering, Xi'an University of Science and Technology, \\ 58 Yanta Road, Xi'an 710054, China \\ ${ }^{1}$ Department of Electronic Engineering, National Chin-Yi University of Technology, \\ No. 57, Sec. 2, Zhongshan Rd., Taiping Dist., Taichung 41170, Taiwan
}

(Received October 3, 2016; accepted April 4, 2017)

Keywords: sensor network, cluster head, routing strategy, energy consumption, lifetime

For performance optimization of a large-scale wireless sensor network (WSN), we developed a new WSN called the WSN with optimal cluster head number (OCHN) and adaptive energy routing strategy (AERS) based on sensor distribution (SD) design, which is referred to as WSNOA. There were three strategies for WSNOA construction. First, a four-ring network model, a heterogeneous energy model of the sensor node, and an energy consumption model of data transmission were designed. Second, the OCHN calculation method was derived on the basis of the energy consumption of each ring and the minimum for the four rings of the WSNOA when sensors were distributed uniformly. Third, the AERS was designed to avoid the non-adaptive energy consumption problem. Compared with the three traditional WSNs, namely, low-energy adaptive clustering hierarchy algorithm (LEACH), stable election protocol (SEP), and ring-based multi-hop clustering routing algorithm (RBMC), the WSNOA was experimentally proved to prolong the WSN lifetime by $25.6 \%$ compared with RBMC and to increase the received data packet quantities by 2.2 , 39.4, and $41.5 \%$ for the sink node compared with the RBMC, SEP, and LEACH, respectively. In addition, the WSNOA was more suitable for the relatively large-scale WSN, especially if the WSN radius is more than $240 \mathrm{~m}$.

\section{Introduction}

With the development of the Internet of Things and 5G technology, data transmission including environmental monitoring and equipment control promote the important role of wireless sensor networks (WSNs) in large-scale wireless communication systems. ${ }^{(1)}$ Wireless sensors usually have small volumes with simple electrical circuits powered by batteries, and the energies of the sensors are definite and limited. ${ }^{(2,3)}$ The problem is that it is difficult or impossible to update the batteries after their energy is used up. ${ }^{(4)}$ Therefore, an important task is to design a WSN that would utilize uniformly the sensor node energy to avoid the phenomenon that a few exhausted sensor nodes will ruin the entire network. ${ }^{(5)}$

The energy consumption of a WSN usually appears during data packet sending and receiving by sensors. ${ }^{(6)}$ The key problem is how to make the sensors which have surplus energy take more tasks and to keep the sensor in the resting state during the no-task period to increase the lifetime *Corresponding author: e-mail: cwy@ncut.edu.tw http://dx.doi.org/10.18494/SAM.2017.1479 
of the WSN. ${ }^{(7)}$ To solve this problem, scientists proposed the low-energy adaptive clustering hierarchy algorithm (LEACH). On the basis of LEACH, researchers developed the modified LEACH (M-LEACH) algorithm, which could save the energy of the WSN on the basis of multiple hops of cluster heads. ${ }^{(8)}$ The algorithm uses the method of dynamic clustering to balance energy consumption. However, it is only suitable for a small WSN because the communication used the single-hop method between the clustering head and the sink node and also the clustering head lose much energy if the communication distance is far.

In addition, a stable election protocol (SEP) was utilized with the two stages of energy for the sensor nodes. ${ }^{(9)}$ Each sensor node was taken as an ordinary node with an initial energy $E_{0}$ or an advanced node with the initial energy $E_{0}(1+\alpha)$. The advanced node possesses more initial energy and has a higher probability to be selected as a clustering head than an ordinary node. This is reasonable because the clustering head needs more energy to transmit its own data and retransmit data from other rings. The WSN with SEP could have a stably longer lifetime. However, it was not suitable for WSN with multiple stages of heterogeneous energy.

A ring-based multi-hop clustering routing algorithm (RBMC) was then developed. ${ }^{(10)}$ It divided the sensor area into several ring regions. The cluster head numbers in the different regions were different. The ring nearest the sink node had more cluster heads because it not only transmitted its own data but also retransmitted data from other rings so that it needed more cluster heads with more energy. Meanwhile, the ring farthest from the sink node had fewer cluster heads because it retransmitted less data from other nodes. Multiple hops were taken for the communication between the cluster heads. However, the lifetime and energy utilization as well as the received data packets of the sink node of a WSN needed to be improved further.

On the basis of these methods, in this study, we developed the sensor distribution (SD) and optimal cluster head number (OCHN) calculation method. Besides, an adaptive energy routing strategy (AERS) was proposed for the energy optimization of the WSN. In Sect. 2, we describe the established models, which include the four-ring model of a WSN and the heterogeneous energy model of the sensor node as well as the energy consumption model of data transmission. In Sect. 3, we designed the SD. In Sect. 4, we derived the OCHN computation method on the basis of energy optimization. In Sect. 5, we present the AERS to avoid the uneven energy consumption problem. It included the definition of the adaptive energy region (AER) of the retransmission cluster head and the routing strategy using the AER. In Sect. 6, we deal with four simulation experiments including lifetimes, cluster head numbers, energy utilization efficiency, and the data packet quantity received by the sink node of WSNOA. The WSNOA is compared with the three traditional WSNs: LEACH, SEP, and RBMC. Finally, in Sect. 7, we provide conclusions.

\section{WSN Model and Node Energy Model}

In a conventional WSN, many sensors were mounted in the nodes of the monitoring region. The four-ring model of WSN, the heterogeneous energy model of sensor nodes, as well as energy consumption model of data transmission were designed as described in the following.

\subsection{Four-ring model of WSN}

A WSN system is shown in Fig. 1. One of the most important parts of a WSN is the monitored region constructed by a sink node and several sensor nodes. It connects to the transmission 


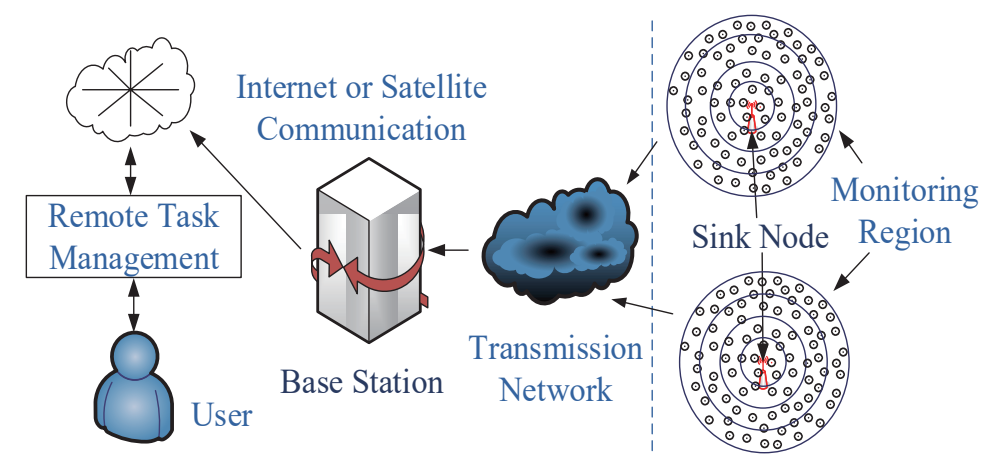

Fig. 1. (Color online) A WSN system.

network, base station, internet or satellite communication, remote task management unit, and user. $^{(11)}$ In this paper, we emphasize the monitoring region in which the black nodes represent ordinate nodes and the red node represents the sink node. The basic structure of a sensor node is shown in Fig. 2. It includes the power supply, processor and memorizer, sensing part, location part, receiver, and transmitter.

The sensors play an important role in a WSN because there are a large number of sensors that build the basic platform of the WSN. The functions of the sensor nodes are to monitor the targets, which include original signal collections, simple data processing or fusion, finite data preservation, and data transmission to the sink node in a single-hop or a multiple-hop. The most important part is the power supply that drives the sensor node. An unpleasant problem is that the energy supply is limited. ${ }^{(12)}$

The sink node is an important node in a WSN because it fulfills the task of whole network data transmission, so it needs much more energy than an ordinary node. The sink node receives the data from sensor nodes in the monitoring region and transmits the data to the base station through the transmission network. ${ }^{(13)}$ The base station utilizes an external network of the Internet or a satellite communications network to send the data to a remote task management center and user. The user analyzes and processes the collected data from the sensors in the terminal. In contrast, the sink node sends requests or control orders to the sensor nodes distributed in the monitored region. In the proactive state, it periodically scans the monitored network and acquires related information from the sensor nodes of the WSN. When the sink node is in the resting state, and it begins to work only when the sensor nodes send a message to the sink node.

Usually, a WSN has an irregular shape determined by the geographical environment. Because the position of the sensor node can be adjusted, it can be assumed that the distribution region of the total $N$ sensors is a circular region with a radius $R$. The advantage of the circular network model is that it is the simplest compared with a square model, polygonal model, or other irregular shape model in the view of topology. In addition, all sensor nodes are relatively closer to the center point. The WSN model with a circular region divided to 4 rings is shown in Fig. 3.

Figure 3(a) is a WSN in which the sensor nodes are generated uniformly and randomly. It is divided into four annular regions with the same space $\delta$ and the same center. Figure 3(b) indicates that the red sensor nodes have used up their energy. The center of the four rings is the sink node. The space between the adjacent rings is $\delta$ meters. The first ring is actually a circle, while the others are annular. 


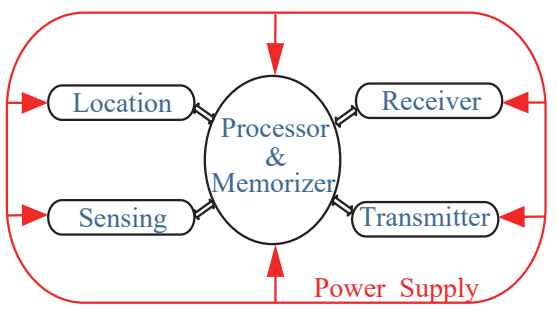

Fig. 2. (Color online) Basic structure of a sensor node.

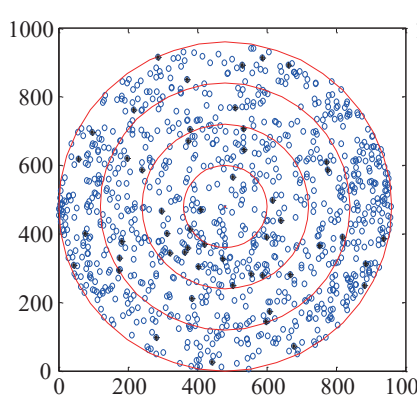

(a)

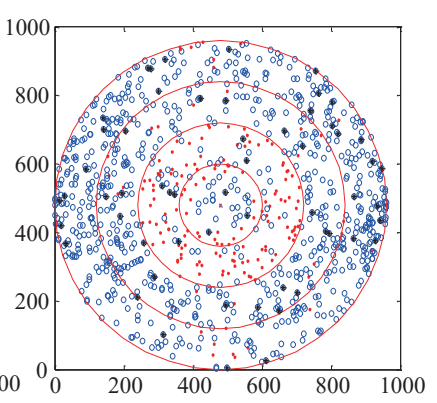

(b)

Fig. 3. (Color online) WSN models of (a) four annular regions with the same space and the same center and (b) a red sensor node with energy exhausted.

\subsection{Heterogeneous energy model of sensor node}

The four rings of the WSN were designed so that the energies of the sensor nodes were in heterogeneous multiple levels. ${ }^{(14)}$ The initial energy interval of each node was $\left[E_{0}, E_{0}(1+\lambda)\right]$. The parameter $E_{0}$ is the lower bound of the initial energy. The parameter $\lambda$ is a positive constant, so the maximum initial energy can be determined. The WSN consists of the set $S$ of $N$ nodes, $S=\left\{s_{i} \mid s_{i}=\left(x_{i}, y_{i}\right), s_{i} \in R^{2}=(4 \delta)^{2}, i=1,2, \ldots, N\right\}$. Therefore, the total initial energy $E_{t}$ of the WSN is

$$
E_{t}=\sum_{i=1}^{N} E_{0}\left(1+\lambda_{i}\right)=E_{0}\left(N+\sum_{i=1}^{N} \lambda_{i}\right)
$$

where $\lambda$ is the energy parameter that determines whether the sensor node energy is heterogeneous or homogeneous. If $\lambda=0$, then the WSN is a homogeneous energy network. Otherwise, the WSN is a heterogeneous energy network.

It can be seen that the initial energy of a heterogeneous energy WSN is equivalent to $\left(N+\sum_{i=1}^{N} \lambda_{i}\right)$ times of the energy of the corresponding homogeneous energy network, where the nodes have the initial energy $E_{0}$.

\subsection{Energy consumption model of data transmission}

The energy cost model of the data transmission of a WSN is shown in Fig. 4. The energy cost of sending $L$ bits of data packets for the distance $d$ includes three parts: the sending cost, the transmission cost, and the receiving cost.

In Fig. 4, $E_{T}$ is the transmission energy power and $E_{R}$ is the received energy power, $\varepsilon$ is the transmission parameters, $d$ is the distance between the sender and the receiver, and $E_{e}$ is the energy cost to send or receive a data bit. The energy power $E_{T}$ consumed by the transmitter is determined as $^{(15)}$

$$
E_{T}(l, d)=\left\{\begin{array}{l}
L \cdot E_{e}+L \cdot \varepsilon_{1} \cdot d^{2} \text { if } d<d_{0} \\
L \cdot E_{e}+L \cdot \varepsilon_{2} \cdot d^{4} \text { if } d \geq d_{0}
\end{array}\right.
$$




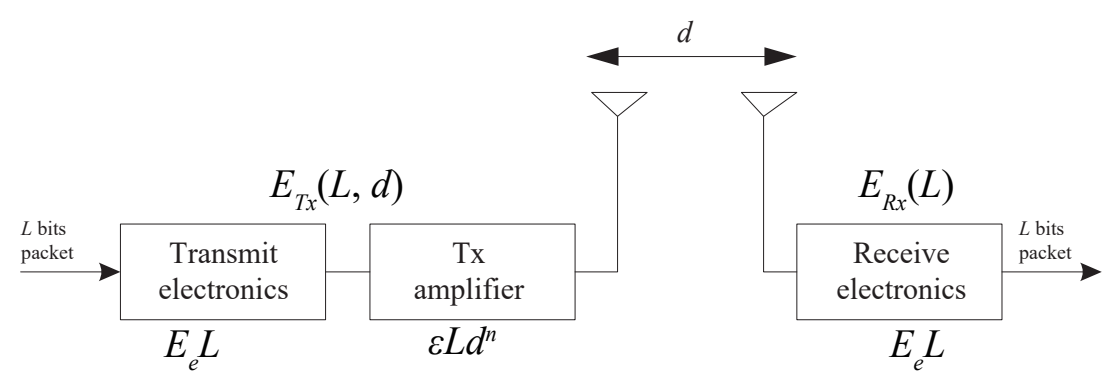

Fig. 4. Energy consumption model of data transmission in WSN.

where $\varepsilon_{1}$ and $\varepsilon_{2}$ are the transmission parameters that distinguish the transmission distance $d$ as being smaller or larger, respectively, than the threshold $d_{0}$.

To find the threshold $d_{0}$, we set the two expressions as equal. That is,

$$
E_{T}(l, d)=L \cdot E_{e}+L \cdot \varepsilon_{1} \cdot d^{2}=L \cdot E_{e}+L \cdot \varepsilon_{2} \cdot d^{4}
$$

As a result, the threshold value $d_{0}$ is found as

$$
d_{0}=\sqrt{\varepsilon_{1} / \varepsilon_{2}}
$$

On the other hand, the energy power $E_{R}$ consumed by the receiver is

$$
E_{R}=L \cdot E_{e},
$$

where $E_{e}$ is the energy cost to receive a data bit. $L$ is the number of data bits.

\section{SD Design}

We designed the sensor nodes to have a uniform distribution in a circular region. The circular region of a WSN is divided into $S$ rings. The radius of each $S$-ring is $\delta, 2 \delta, \ldots, S \delta$, respectively.

The total number of nodes in the WSN is $N$. The number of nodes in each ring is $N_{1}, N_{2}, \ldots, N_{S}$. The areas $a_{1}, a_{2}, \ldots, N_{S}$ of the $S$ rings are as follows:

$$
\begin{gathered}
a_{1}=\pi \delta^{2} / 2, \\
a_{2}=\pi(2 \delta)^{2} / 2-\pi \delta^{2} / 2=3 \pi \delta^{2} / 2=3 a_{1}, \\
a_{3}=\pi(3 \delta)^{2} / 2-\pi(2 \delta)^{2} / 2=5 \pi \delta^{2} / 2=5 a_{1}, \\
a_{4}=\pi(4 \delta)^{2} / 2-\pi(3 \delta)^{2} / 2=7 \pi \delta^{2} / 2=7 a_{1}, \\
a_{k}=\pi(k \delta)^{2} / 2-\pi[(k-1) \delta]^{2} / 2=(2 k-1) \pi \delta^{2} / 2=(2 k-1) \cdot a_{1}, \\
a_{S}=\pi(S \delta)^{2} / 2-\pi[(S-1) \delta]^{2} / 2=(2 S-1) \pi \delta^{2} / 2=(2 S-1) \cdot a_{1},
\end{gathered}
$$


where, $k=1,2, \ldots, S$. The term $\delta$ is the space of the rings distributed uniformly.

Because the sensor nodes are distributed uniformly, the numbers $N_{1}, N_{2}, \ldots, N_{S}$ of the sensor nodes in all the rings are proportional to their areas.

$$
\begin{gathered}
N_{1}=N \cdot \frac{\pi \delta^{2} / 2}{\pi(S \delta)^{2} / 2}=N / S^{2}, \\
N_{2}=3 N_{1}=3 N / S^{2}, \\
N_{3}=5 N_{1}=5 N / S^{2}, \\
N_{4}=7 N_{1}=7 N / S^{2}, \\
N_{k}=(2 k-1) N_{1}=(2 k-1) N / S^{2}, \\
N_{S}=(2 S-1) N_{1}=(2 S-1) N / S^{2},
\end{gathered}
$$

where $S$ is the total number of rings in the monitored region.

If $S=1$, then the number $N_{S 1}$ of sensor nodes in the ring according to Eq. (17) is

$$
N_{S 1}=N .
$$

If $S=2$, then the number $N_{S k}$ of sensor nodes in the ring $k$ according to Eqs. (16) and (17) is

$$
\left\{\begin{array}{l}
N_{S 1}=N / 4 \\
N_{S 2}=3 N / 4
\end{array}\right.
$$

If $S=3$, then the number $N_{S k}$ of sensor nodes in the ring $k$ according to Eqs. (16) and (17) is

$$
\left\{\begin{array}{l}
N_{S 1}=N / 9 \\
N_{S 2}=3 N / 9 \\
N_{S 3}=5 N / 9
\end{array}\right.
$$

If $S=4$, then the number $N_{S k}$ of sensor nodes in the ring $k$ according to Eqs. (16) and (17) is

$$
\left\{\begin{array}{l}
N_{S 1}=N / 16 \\
N_{S 2}=3 N / 16 \\
N_{S 3}=5 N / 16 \\
N_{S 4}=7 N / 16
\end{array}\right.
$$

Equations (18)-(21) are the number of sensors of each ring for a WSN with 1-4 rings, respectively. By combining this analysis with the constraint of uniform distribution, the SD design was completed. 


\section{Derivation of OCHN Based on Ring Energy Optimization}

To improve the energy efficiency of the WSN, we extended the lifetime and increased the data quantity received by the sink node; the average energy consumption of the cluster heads of each ring is designed to be equal and minimum, so the normal communication of all the sensors is ensured simultaneously.

We assume that the numbers of cluster heads of the first ring and ring $k$ are $m_{1}$ and $m_{k}$, respectively. Meanwhile, the total number of nodes within ring $k$ is $N_{k}$. Then, the optimal number of cluster heads ${ }^{(16)}$ within ring $k, m_{k o}$, is

$$
m_{k o}=\sqrt{\frac{N_{k}}{2}} \frac{\delta}{\sqrt{E\left[d_{c h k}^{2}\right]}},
$$

where $k$ is the ring number from inside to outside, $k=1,2, \ldots, S . E\left[d_{c h k}^{2}\right]$ is the squared expectation of the distance from the cluster head to the sink node in ring $k$. That is,

$$
E\left[d_{c h k}^{2}\right]=\frac{k^{2}+(k-1)^{2}}{2} \delta^{2}
$$

Substituting Eqs. (16) and (23) into Eq. (22), we obtain the optimal number of cluster heads of WSN with $S$ ring, $m_{S k o}$, as

$$
m_{S k o}=\sqrt{\frac{(2 k-1) N}{\left(2 k^{2}-2 k+1\right)}} / S,
$$

where $S$ is the total number of rings, and $k$ is the ring number from inside to outside, $k=1,2, \ldots, S$. $N$ is the total number of sensor nodes in the WSN.

The OCHN was obtained using Eq. (24). If $S=1$ and $k=1$ were substituted into Eq. (24), then

$$
M_{S k o}=\sqrt{N}, S=1 \& k=1 .
$$

Similarly, we have

$$
\begin{aligned}
& m_{\text {Sko }}=\left\{\begin{array}{l}
\sqrt{N} / 2, S=2 \& k=1 \\
\sqrt{3 N / 5} / 2, S=2 \& k=2
\end{array},\right. \\
& m_{\text {Sko }}=\left\{\begin{array}{l}
\sqrt{N} / 3, S=3 \& k=1 \\
\sqrt{3 N / 5} / 3, S=3 \& k=2 \\
\sqrt{5 N / 13} / 3, S=3 \& k=3
\end{array}\right. \\
& m_{\text {Sko }}=\left\{\begin{array}{l}
\sqrt{N} / 4, S=4 \& k=1 \\
\sqrt{3 N / 5} / 4, S=4 \& k=2 \\
\sqrt{5 N / 13} / 4, S=4 \& k=3 \\
\sqrt{7 N / 25} / 4, S=4 \& k=4
\end{array}\right.
\end{aligned}
$$


Thus, we obtained the result of the OCHN from the above derivation process, that is, the areas $a_{1}, a_{2}, \ldots, N_{S}$ of the $S$ rings would increase by odd factors from the inside ring to the outside ring. Meanwhile, the sensor node number of each ring increased similarly to the areas because the sensor nodes are distributed uniformly. However, the optimal cluster head number decreased nonlinearly from the inside ring to the outside ring based on the energy minimization of each ring and equality because the cluster heads of the inside ring not only collect the data from non-cluster nodes within cluster itself and send the data to the sink node, but they also receive data from other rings and retransmit the data to sink node. Therefore, the derived OCHNs of the inside rings are larger than those of the outside rings, which could improve the energy efficiency of WSN.

\section{New WSN Based on SD and OCHN as well as AERS (WSNOA)}

The WSNOA work scheme is shown in Fig. 5. It includes the WSN start, radius determination, OCHN calculation, the routing determine based on AERS, and finally the collection and transmission of sensor information. The WSN radius and sensor node number are determined at the beginning of a WSN design. The OCHN was derived above. So we would define the AER and propose the AERS below. Figure 6 is the design scheme of the WSNOA.

\subsection{Definition of AER}

Assuming that $S_{k}$ is a cluster head node in ring $k$, we define the radius $r$ of AER for $S_{k}$ to find the next cluster head node that receives data from $S_{k}$.

$$
r=\left[1+\frac{d\left(S_{k}, \text { Sink }\right)-\delta}{d_{\max }-d_{\min }} \cdot \eta\right] \delta
$$

where $\eta$ is a positive decimal that regulates the radius $\mathrm{r}$ of the available region. The terms $d_{\max }$ and $d_{m i n}$ represent respectively the maximum and minimum distances between the cluster head node $S_{k}$ and the sink node. The term $d\left(S_{k}, S\right.$ ink $)$ represents the distance between the cluster head node $S_{k}$ and the sink node, and $\delta$ is the radius difference between the neighboring rings.

The radius $r$ is a positive number which is greater than the radius difference $\delta$. If the distance between the cluster head node $S_{k}$ and the sink node is large, $r$ will also be relatively larger.

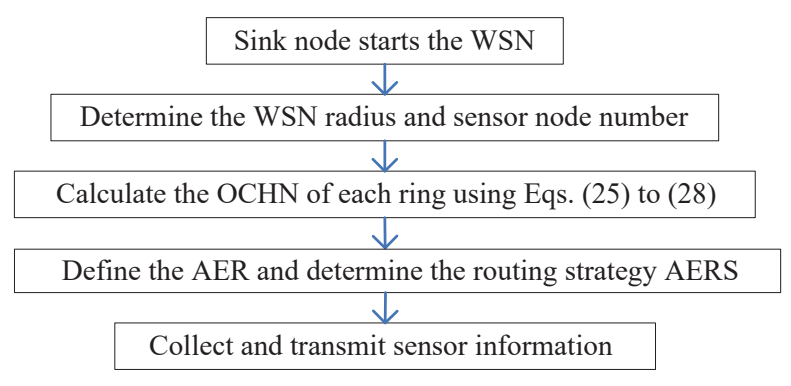

Fig. 5. WSNOA work scheme. 


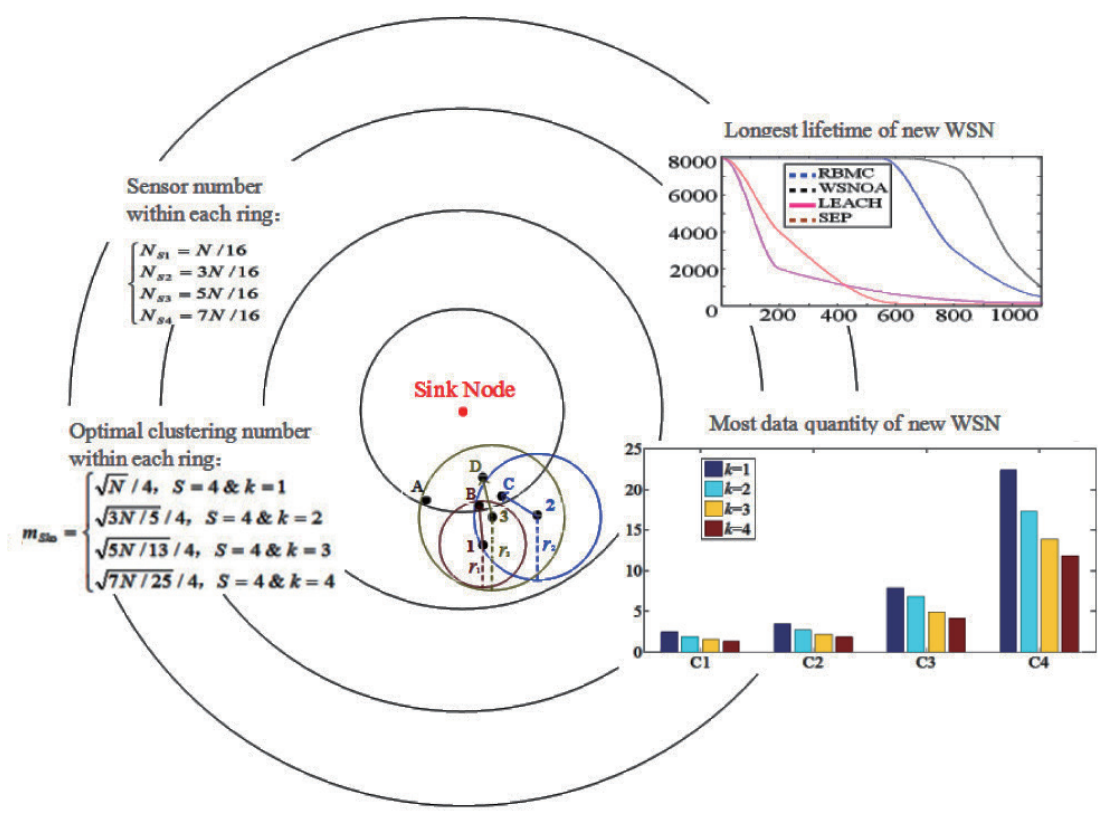

Fig. 6. (Color online) Design scheme of the WSNOA.

\subsection{AERS}

The ordinary sensor nodes except for the first ring acquire local physical information and send their data to the corresponding cluster head nodes. The cluster head nodes collect the data from the ordinary sensor nodes and form a data packet. ${ }^{(17,18)}$ Then, the cluster head node must find another cluster head in the next ring to receive and retransmit the data packet. The traditional multiple-hop routing strategy chooses the nearest cluster head node in the next ring. Although this method saves the energy of the sender, the receiver cluster head usually performs multiple tasks; simultaneously, the cluster head is completely free. Thus, the non-balanced energy consumption problem occurs. To solve this problem, we present the AERS.

The traditional multiple-hop routing strategy is shown in Fig. 7. The cluster head node of the outside ring selects the nearest cluster head node inside the ring as its forwarding cluster head. All the nodes in region 1 are nearest to the cluster head 1, so all the cluster head nodes in region 1 choose cluster head 1 as their forwarding cluster head. By observing the cluster head of the inside ring, it can be found that the cluster heads 1 and 3 as well as 4 perform all the retransmission tasks of the outside ring. However, the cluster heads 2 and 5 are completely free. As a result, the cluster heads 2 and 5 have surplus energy. The cluster heads 1 and 3 as well as 4 use up their energy quickly, so the lifetime of the whole network is affected.

To target this problem, that is that non-uniform energies affect the lifetime of the whole network, in this paper, we present the AERS and explain it using Fig. 8.

Rule 1: The cluster head within the available region of the smallest radius has the highest priority of being selected as the current forwarding cluster head.

Rule 2: The cluster head within the available region, which had been selected as the former forwarding cluster head, has a low-level priority of being selected as the current forwarding cluster head. 


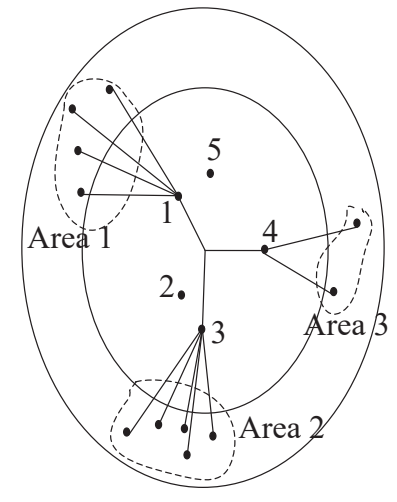

Fig. 7. Traditional multiple-hop routing strategy.

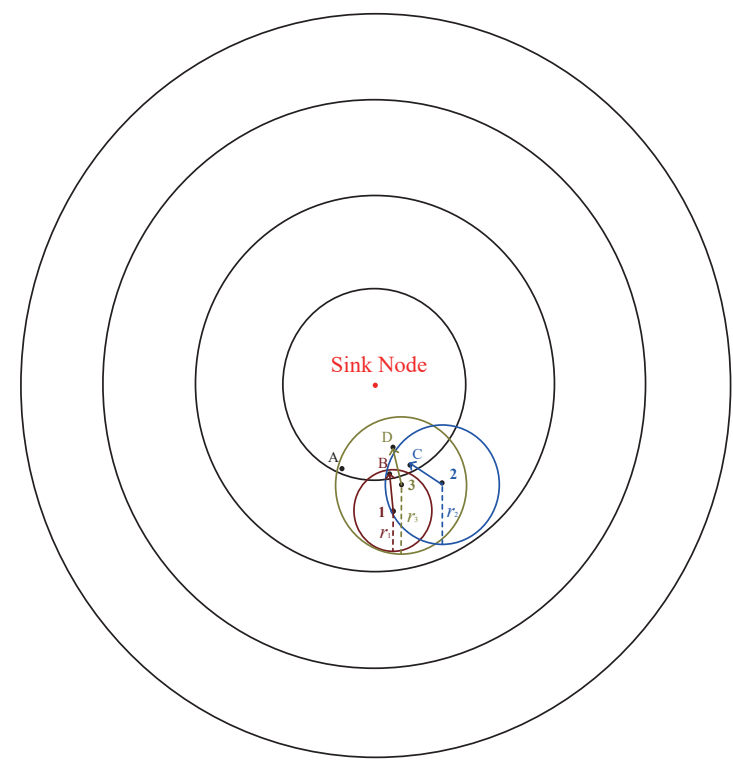

Fig. 8. (Color online) AERS.

Rule 3: If there is no a cluster head node in the available region, adjust the parameter of Eq. (29) to enlarge the radius of the available region, until there is a cluster head node in the available region.

In the AERS in Fig. 7, the cluster head nodes 1 and 2 as well as 3 are in the outside ring. Their available region radiuses are $r_{1}, r_{2}$, and $r_{3}$, respectively, $r_{1}<r_{2}<r_{3}$.

The AER of node 1 only contains node $\mathrm{B}$, so node $\mathrm{B}$ will serve as the forwarding cluster node for node 1. The AER of node 2 contains nodes B and C. Because node B had been chosen for node 1, node $\mathrm{C}$ will be determined as the forwarding cluster head for node 2. The AER of node 3 contains nodes A-D. Nodes B and C were chosen in the previous two steps, and node D is nearer to node 3. Therefore, node $\mathrm{D}$ will be determined as the forwarding cluster head for node 3 according to AERS.

\section{Simulation and Analysis}

We designed simulation experiments. (a) $R=100 \mathrm{~m}, N=100, S=1, \lambda=0$. (b) $R=100 \mathrm{~m}, N=$ $100, S=1, \lambda \neq 0$. (c) $R=160 \mathrm{~m}, N=200, S=2, \lambda=0$. (d) $R=160 \mathrm{~m}, N=200, S=2, \lambda \neq 0$. (e) $R$ $=240 \mathrm{~m}, \mathrm{~N}=1000, S=3, \lambda=0$. (f) $R=240 \mathrm{~m}, N=1000, S=3, \lambda \neq 0$. (g) $R=720 \mathrm{~m}, N=8000, S$ $=4, \lambda=0$. (h) $R=720 \mathrm{~m}, N=8000, S=4, \lambda \neq 0$. Here, $R$ is the radius of the sensor area, $N$ is the total number of sensors of the WSN, and $S$ is the number of rings of the WSN. The heterogeneous energy parameter $\lambda \neq 0$, and the homogeneous energy parameter $\lambda=0$.

\subsection{Simulation environment}

The simulation environment system used a computer with Intel(R), Core(TM) i3-2130 CPU 3.4 GHz. The operating system was Windows XP. The software platform was Matlab.

The environmental parameters of the simulation are shown in Tables 1 and 2. In Table 1, "Ho" represents the homogeneous energy $\lambda=0$, and "He" represents the heterogeneous energy $\lambda \neq 0$. 
Table 1

Four simulation cases and their initial energies.

\begin{tabular}{lccccc}
\hline Case No. & $\begin{array}{c}\text { Energy } \\
\text { type }\end{array}$ & Radius & $\begin{array}{c}\text { Number } \\
\text { of nodes }\end{array}$ & $\begin{array}{c}\text { Number } \\
\text { of rings }\end{array}$ & Energy \\
\hline 1 & Ho & $100 \mathrm{~m}$ & 100 & 1 & $1 \mathrm{~J}$ \\
& $\mathrm{He}$ & $100 \mathrm{~m}$ & 100 & 1 & {$[1,2] \mathrm{J}$} \\
2 & $\mathrm{Ho}$ & $160 \mathrm{~m}$ & 200 & 2 & $2 \mathrm{~J}$ \\
& $\mathrm{He}$ & $160 \mathrm{~m}$ & 200 & 2 & {$[2,2.5,3,3.5] \mathrm{J}$} \\
3 & $\mathrm{Ho}$ & $240 \mathrm{~m}$ & 1000 & 3 & $5 \mathrm{~J}$ \\
& $\mathrm{He}$ & $240 \mathrm{~m}$ & 1000 & 3 & {$[6,6.5,7,7.5,8,9.5,11] \mathrm{J}$} \\
4 & $\mathrm{Ho}$ & $720 \mathrm{~m}$ & 8000 & 4 & $8 \mathrm{~J}$ \\
& $\mathrm{He}$ & $720 \mathrm{~m}$ & 8000 & 4 & {$[8,8.5,9,9.5,10,10.5,11,11.5,12] \mathrm{J}$} \\
\hline
\end{tabular}

Table 2

Sink node position and transmission energy parameter.

\begin{tabular}{lc}
\hline Parameter & Value \\
\hline Sink node position & $(0,0)$ \\
$E_{T}, E_{R}$ & $5.0 \times 10^{-8} \mathrm{~J} / \mathrm{bit}$ \\
$E_{e}$ & $5.0 \times 10^{-8} \mathrm{~J} / \mathrm{bit}$ \\
$\varepsilon_{1}$ & $1.0 \times 10^{-11} \mathrm{~J} / \mathrm{bit}$ \\
$\varepsilon_{2}$ & $1.3 \times 10^{-15} \mathrm{~J} / \mathrm{bit}$ \\
\hline
\end{tabular}

\subsection{Evaluation and simulation comparison}

In this section, we evaluate the new WSNOA by simulating lifetime, number of cluster heads, energy utilization, and the total quantity of data packets received by the sink node. The simulation results are compared with the three traditional methods, namely, LEACH, SEP and RBMC.

\section{(1) Lifetime}

The relationship between the number of remaining available sensor nodes of the WSN and the data collection cycle number is shown in Fig. 9. The horizontal axis represents the data collection cycle number, and the vertical axis represents the number of remaining available nodes. We check the WSN lifetime by inspecting the data collection cycle number when the first node dies.

In case 1 of Figs. 9 (a) and 9(b), $R=100 \mathrm{~m}, N=100, S=1$. However, $\lambda=0$ and $\lambda \neq 0$, respectively, for Figs. 9(a) and 9(b). This means that WSN is a homogeneous energy network and a heterogeneous energy network in Figs. 9(a) and 9(b), respectively. It can be seen that the WSNOA had a short lifetime if the WSNOA is a small network compared with the traditional LEACH network and SEP network.

In case 2 of Fig. 9 (c), $R=160 \mathrm{~m}, N=200, S=2$, and $\lambda=0$. It can be seen that the lifetime of WSNOA is 983 rings. Meanwhile, the lifetimes of RBMC, SEP, and LEACH are 814, 446, and 412 rings. Thus, the lifetime of WSNOA is improved by $17.19,54.63$, and $58.19 \%$, respectively. In case 2 of Fig. 9(d), $R=160 \mathrm{~m}, N=200, S=2$, and $\lambda \neq 0$. The lifetime of WSNOA is 1214 rings. Meanwhile, the lifetimes of RBMC, SEP, and LEACH are 1143, 627, and 884 rings, respectively. Thus, the lifetime of WSNOA is improved by $5.85,48.35$, and $27.18 \%$, respectively.

In case 3 of Fig. 9(e), $R=240 \mathrm{~m}, N=1000, S=3$, and $\lambda=0$. The lifetime of WSNOA is almost the same as that of RBMC, and better than those of LEACH and SEP. In case 6 of Fig. 9(f), $R=240 \mathrm{~m}$, 


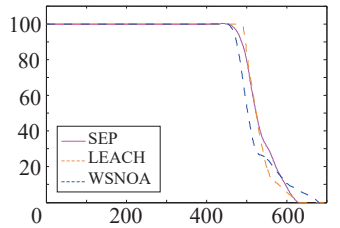

(a)

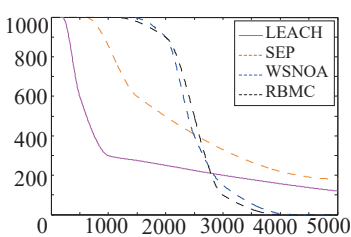

(e)

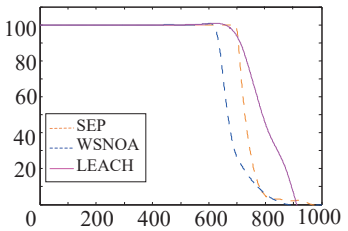

(b)

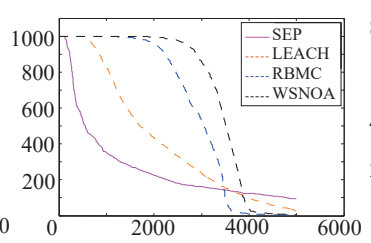

(f)

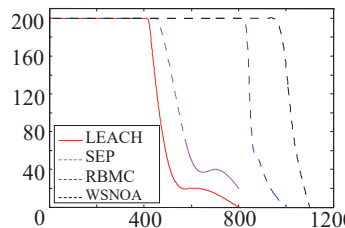

(c)

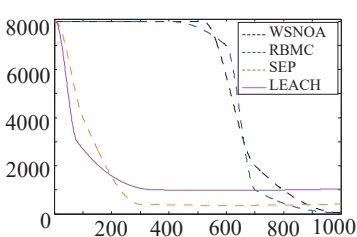

(g)

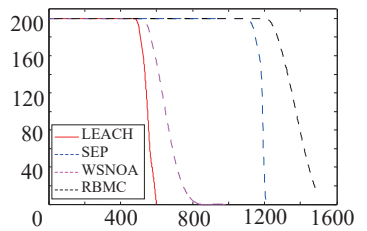

(d)

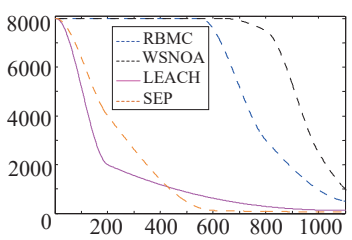

(h)

Fig. 9. (Color online) Relationships between the number of remaining available sensor nodes and the data collection cycle number of (a) Case 1 with $\lambda=0$, (b) Case 1 with $\lambda \neq 0$, (c) Case 2 with $\lambda=0$, (d) Case 2 with $\lambda \neq 0$, (e) Case 3 with $\lambda=0$, (f) Case 3 with $\lambda \neq 0$, (g) Case 4 with $\lambda=0$, and (h) Case 4 with $\lambda \neq 0$.

$N=1000, S=3$, and $\lambda \neq 0$. The lifetime of WSNOA is 2834 rings. Meanwhile, the lifetimes of RBMC, SEP, and LEACH are 1953, 734, and 267 rings, respectively. Compared with RBMC, SEP and $\mathrm{LEACH}$, the lifetime of WSNOA is prolonged by 31.0, 74.1, and $90.6 \%$, respectively. Thus, the superiority of WSNOA is reflected clearly as the WSNOA size increases. In contrast, RBMC, SEP, and LEACH have relatively unsatisfactory lifetimes with the large-scale network.

In case 4 of Figs. $9(\mathrm{~g})$ and $9(\mathrm{~h}), R=720 \mathrm{~m}, N=8000$, and $S=4$. It can be seen that both the homogeneous and heterogeneous energy sensor nodes start to die in the 4th cycles of the SEP and LEACH networks. The death speed of nodes accelerates before 200 cycles are achieved. The nodes stop dying and become stable when 300 cycles are achieved because the network region is sufficiently large and the far cluster head nodes cannot send a message to the sink node. Thus, these nodes have lost the environment sensing functions. That is, the WSN is unavailable. In Fig. 9(h), $R=720 \mathrm{~m}, N=8000, S=4$, and $\lambda \neq 0$. The lifetime of the WSNOA is 793 rings. Meanwhile, the lifetime of the RBMC is 590 rings. This means that the lifetime of the WSNOA increases by 25.6\%. In addition, SEP and LEACH are unsuitable for the large WSN. Although the lifetime of the WSNOA is shorter than case 2 of $R=240 \mathrm{~m}, N=1000$, and $S=3$, the WSNOA also possesses a much longer lifetime than SEP and LEACH. The lifetime comparisons of the four cases are presented in Table 3.

According to Fig. 9 and Table 3, the results indicate that the WSNOA has a longer lifetime for a large WSN than SEP, LEACH and RBMC. Meanwhile, SEP and LEACH are only suitable for a small WSN. In addition, RBMC is better than SEP and LEACH.

(2) Optimal number of cluster heads

According to the derived OCHN of Eq. (24) or the detailed forms of Eqs. (25)-(28), we draw the optimal number of cluster heads for the four cases in Fig. 10. The term $\mathrm{C} 1$ represents $N=100$, C2 represents $N=200, \mathrm{C} 3$ represents $N=1000, \mathrm{C} 4$ represents $N=8000$, and $k$ is the number of rings. It can be seen that the optimal number of cluster heads increases as the total number of sensor nodes increases. In addition, the optimal number of cluster heads of each ring decreases from the inside ring to the outside ring. 
Table 3

Comparison of lifetimes.

\begin{tabular}{|c|c|c|c|c|c|}
\hline Case No. & Parameters & LEACH & SEP & RBMC & WSNOA \\
\hline \multirow{2}{*}{1} & $R=100 \mathrm{~m}, N=100, S=1, \lambda=0$ & Longer & Medium & Null & Shorter \\
\hline & $R=100 \mathrm{~m}, N=100, S=1, \lambda \neq 0$ & Medium & Longer & Null & Shorter \\
\hline \multirow{2}{*}{2} & $R=160 \mathrm{~m}, N=200, S=2, \lambda=0$ & Shortest & Shorter & Longer & Longest \\
\hline & $R=160 \mathrm{~m}, N=200, S=2, \lambda \neq 0$ & Shortest & Shorter & Longer & Longest \\
\hline \multirow{2}{*}{3} & $R=240 \mathrm{~m}, N=1000, S=3, \lambda=0$ & Shortest & Shorter & Longest & Longer \\
\hline & $R=240 \mathrm{~m}, N=1000, S=3, \lambda \neq 0$ & Shortest & Shorter & Longer & Longest \\
\hline \multirow{2}{*}{4} & $R=720 \mathrm{~m}, N=8000, S=4, \lambda=0$ & Shortest & Shorter & Longer & Longest \\
\hline & $R=720 \mathrm{~m}, N=8000, S=4, \lambda \neq 0$ & Shortest & Shorter & Longer & Longest \\
\hline
\end{tabular}

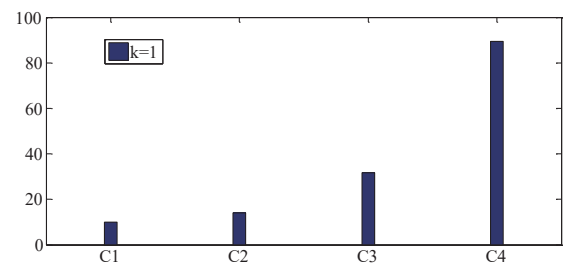

(a)

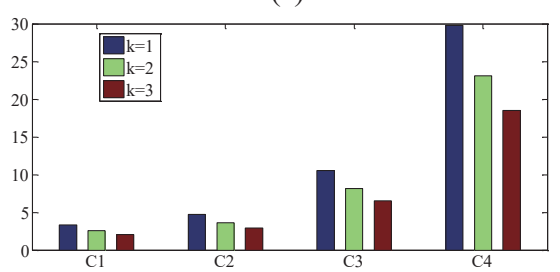

(c)

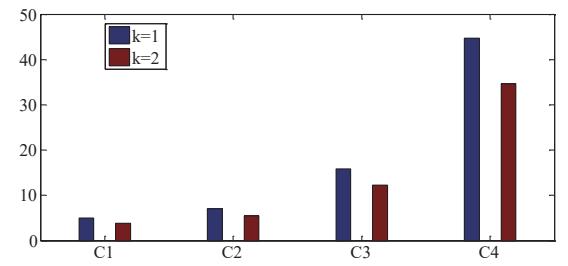

(b)

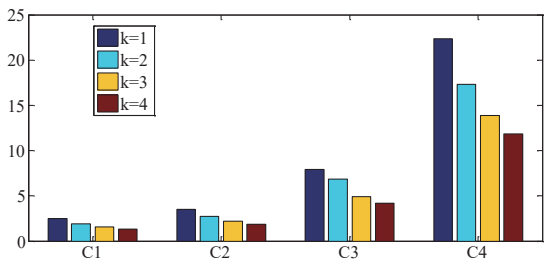

(d)

Fig. 10. (Color online) Optimal numbers of cluster heads for (a) $S=1$, (b) $S=2$, (c) $S=3$, and (d) $S=4$.

The relationship between the optimal number of cluster heads and the data collection cycle number is shown in Fig. 11 It can be seen from Figs. 11(a) and 11(b) that the optimal number of cluster heads was about 11 for all WSNOA, SEP, and LEACH. The optimal numbers are relatively stable before the first sensor node dies. After the lifetime is over, the optimal number of cluster heads becomes unstable with the increasing number of simulation cycles because the total number of remaining sensor nodes is unstable and the energy consumption rate increases rapidly. In addition, the optimal number of cluster heads of the WSNOA is less than the number of cluster heads in the others, as shown in Figs. 11(a)-11(c). The comparison of cluster head numbers is shown in Table 4. According to Fig. 11 and Table 4, the WSNOA had fewer but more stable cluster heads than SEP, LEACH and RBMC.

(3) Total energy consumption of sensor network

For the four cases, the relationship between the total energy consumption of the WSN and the data collection cycle number are simulated in Fig. 12. It can be seen that the energy consumption rate of the WSNOA is clearly slower than that of LEACH and SEP, except in case 1 . Therefore, the WSNOA decreases the energy consumption rate in the larger-scale WSN, and the energy utilization efficiency of the WSN is increased. A comparison of total energy consumption is given in Table 5. 


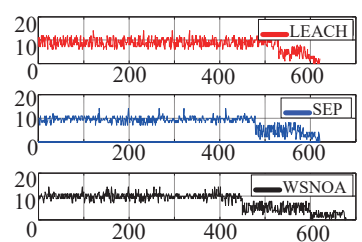

(a)

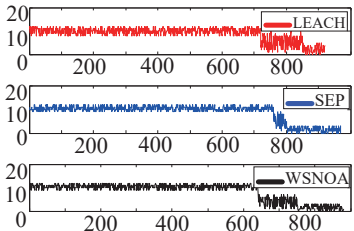

(b)

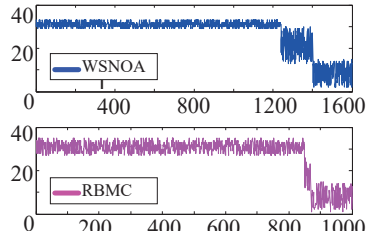

(c)

Fig. 11. (Color online) Relationships between the number of cluster heads and the data collection cycle number of (a) $R=100 \mathrm{~m}, N=100, S=1$, and $\lambda=0$; (b) $R=100 \mathrm{~m}, N=100, S=1$, and $\lambda \neq 0$; (c) $R=160 \mathrm{~m}, N=200, S=2$, and $\lambda \neq 0$.

Table 4

Comparison of number of cluster heads.

\begin{tabular}{lcccc}
\hline Parameters & LEACH & SEP & RBMC & WSNOA \\
\hline$R=100 \mathrm{~m}, N=100, S=1, \lambda=0$ & More & Medium & Null & Less \\
$R=100 \mathrm{~m}, N=100, S=1, \lambda \neq 0$ & Medium & More & Null & Less \\
$R=160 \mathrm{~m}, N=200, S=2, \lambda \neq 0$ & Null & Null & More & Less \\
\hline
\end{tabular}

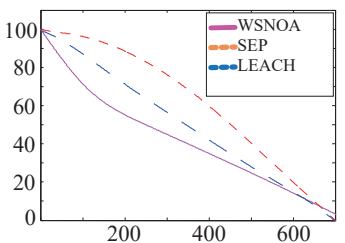

(a)

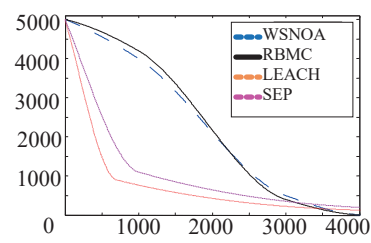

(e)

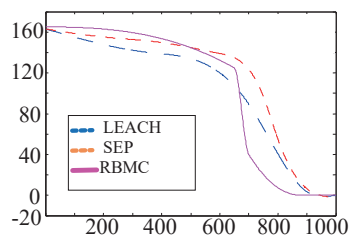

(b)

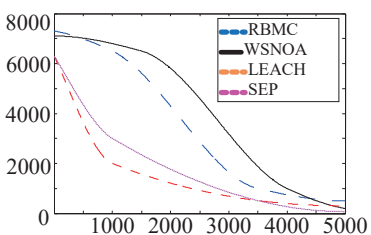

(f)

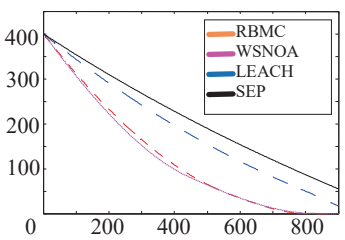

(c)

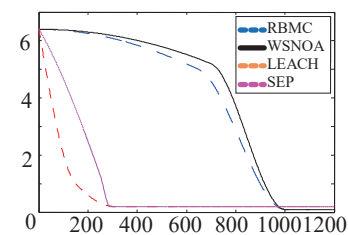

(g)

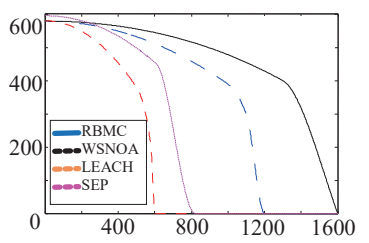

(d)

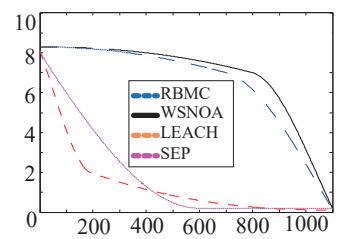

(h)

Fig. 12. (Color online) Relationships between the total energy consumption and the data collection cycle number of (a) Case 1 with $\lambda=0$, (b) Case 1 with $\lambda \neq 0$, (c) Case 2 with $\lambda=0$, (d) Case 2 with $\lambda \neq 0$, (e) Case 3 with $\lambda=0$, (f) Case 3 with $\lambda \neq 0$, (g) Case 4 with $\lambda=0$, and (h) Case 4 with $\lambda \neq 0$.

Table 5

Comparison of total energy consumption.

\begin{tabular}{|c|c|c|c|c|c|}
\hline Case No. & Parameters & LEACH & SEP & RBMC & WSNOA \\
\hline \multirow{2}{*}{1} & $R=100 \mathrm{~m}, N=100, S=1, \lambda=0$ & Medium & High & Null & Lower \\
\hline & $R=100 \mathrm{~m}, N=100, S=1, \lambda \neq 0$ & Lower & Medium & Null & Higher \\
\hline \multirow{2}{*}{2} & $R=160 \mathrm{~m}, N=200, S=2, \lambda=0$ & Lower & Lowest & Highest & Highest \\
\hline & $R=160 \mathrm{~m}, N=200, S=2, \lambda \neq 0$ & Lower & Lowest & Higher & Highest \\
\hline \multirow{2}{*}{3} & $R=240 \mathrm{~m}, N=1000, S=3, \lambda=0$ & Lower & Lowest & Higher & Highest \\
\hline & $R=240 \mathrm{~m}, N=1000, S=3, \lambda \neq 0$ & Lower & Lowest & Highest & Higher \\
\hline \multirow{2}{*}{4} & $R=720 \mathrm{~m}, N=8000, S=4, \lambda=0$ & Lower & Lowest & Higher & Highest \\
\hline & $R=720 \mathrm{~m}, N=8000, S=4, \lambda \neq 0$ & Lower & Lowest & Higher & Highest \\
\hline
\end{tabular}


According to Fig. 12 and Table 5, WSNOA can utilize the node energies in balance to prolong the lifetime of a large-scale WSN compared with SEP, LEACH, and RBMC. Meanwhile, SEP and LEACH are only suitable for the small-scale WSN. In addition, RBMC is better than SEP and LEACH for energy utilization.

(4) Received data packet quantity of sink node

The relationship between the received data packet quantity of the sink node and the data collection cycle number are shown in Fig. 13. Table 6 compares the numbers of received data packets of the sink nodes.

It can be seen that the WSNOA received more data packets than SEP and LEACH in the four cases. For example, the sink node of the WSNOA received more data packets in case 4 than that of RBMC, SEP, and LEACH by $2.2,39.4$, and $41.5 \%$, respectively. Besides, on the base of the received data packet quantity of the sink nodes, the sensor nodes with homogeneous energy are more suitable for a small WSN than those with heterogeneous energy in case 1. According to Fig. 13 and Table 6, the sink node of the WSNOA can receive many more data packets than those of LEACH, SEP, and RBMC.

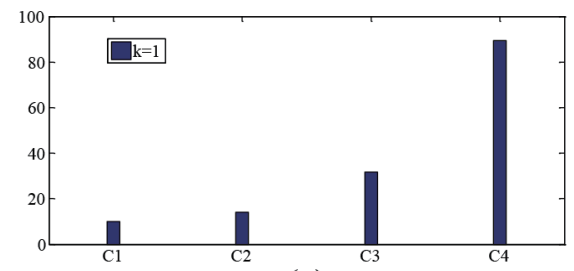

(a)

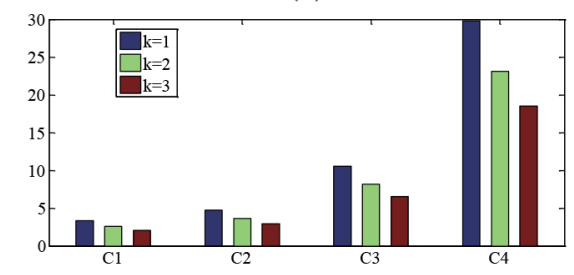

(c)

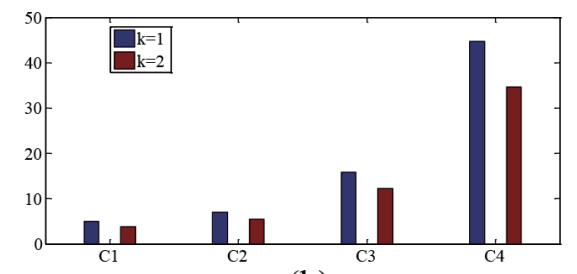

(b)

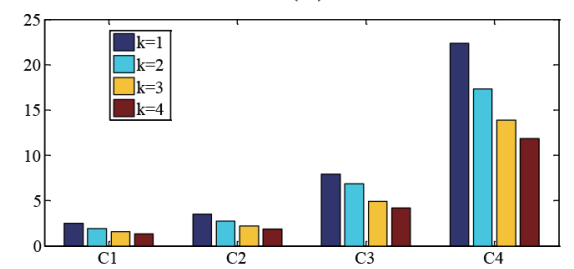

(d)

Fig. 13. (Color online) Relationship between the received data packet quantity of the sink node and the data collection cycle number of (a) Case 1 with $\lambda=0$, (b) Case 1 with $\lambda \neq 0$, (c) Case 2 with $\lambda=0$, (d) Case 2 with $\lambda \neq 0$, (e) Case 3 with $\lambda=0$, (f) Case 3 with $\lambda \neq 0$, (g) Case 4 with $\lambda=0$, and (h) Case 4 with $\lambda \neq 0$.

Table 6

Comparison of number of received data packets of sink nodes.

\begin{tabular}{lccccc}
\hline Case No. & Experiment & LEACH & SEP & RBMC & WSNOA \\
\hline 1 & $R=100 \mathrm{~m}, N=100, S=1, \lambda=0$ & Least & Less & Null & Much more \\
2 & $R=100 \mathrm{~m}, N=100, S=1, \lambda \neq 0$ & Least & Less & Null & Much more \\
3 & $R=160 \mathrm{~m}, N=200, S=2, \lambda=0$ & Least & Less & More & Much more \\
4 & $R=160 \mathrm{~m}, N=200, S=2, \lambda \neq 0$ & Least & Less & More & Much more \\
5 & $R=240 \mathrm{~m}, N=1000, S=3, \lambda=0$ & Least & Less & More & Much more \\
6 & $R=240 \mathrm{~m}, N=1000, S=3, \lambda \neq 0$ & Least & Less & More & Much more \\
7 & $R=720 \mathrm{~m}, N=8000, S=4, \lambda=0$ & Least & Less & More & Much more \\
8 & $R=720 \mathrm{~m}, N=8000, S=4, \lambda \neq 0$ & Least & Less & More & Much more \\
\hline
\end{tabular}




\section{Conclusions}

To overcome the problem that non-adaptive energy consumption impacts the lifetime of a WSN, a new kind of WSN was proposed, designated by WSNOA. There were three strategies for WSNOA construction. First, a four-ring network model, a heterogeneous energy model of the sensor nodes, and an energy consumption model of data transmission were designed. Second, the OCHN calculation method was derived on the basis of minimum and equal ring energy consumption in a wireless network where sensors are distributed uniformly. Third, the AERS was presented to avoid the non-adaptive energy consumption problem. Compared with the three traditional WSNs with LEACH, SEP, and RBMC methods, four simulations proved that the new WSNOA performed better, namely, had a longer network lifetime, fewer but more stable number of cluster heads, improved network energy efficiency, and more data received by the sink node. For the case of $R=720 \mathrm{~m}, N=8000, S=4$, and $\lambda \neq 0$, WSNOA prolongs the lifetime by $25.6 \%$ compared with RBMC. Meanwhile, SEP and LEACH are unsuitable for the large scale of a WSN. In addition, WSNOA increases the quantities of received data packets of the sink node by $2.2,39.4$, and $41.5 \%$, respectively, compared with RBMC, SEP, and LEACH. In addition, WSNOA is more suitable for a relatively large-scale WSN, especially if the WSN radius is more than $240 \mathrm{~m}$.

\section{Acknowledgments}

This research was sponsored by the Natural Science Foundation of China (51405381), the Key Scientific and Technological Project of Shaanxi Province (2016GY-040), and the Science Foundation of Xi'an University of Science and Technology (104-6319900001).

\section{References}

M. Agiwal, A. Roy, and N. Saxena: IEEE Commun. Surv. Tutorials 18 (2016) 1617.

2 C. S. Nam, Y. S. Han, and D. R. Shin: Sensors 11 (2011) 2875.

3 A. Abahazem, H. Guedah, N. Merbahi, M. Yousfi, O. Eichwald, and A. Ihla: Mater. Today 2 (2015) 4694.

4 N. Terasaki: Sens. Mater. 28 (2016) 827.

5 H. Li and J. Liu: Int. J. Wireless Inf. Networks 23 (2016) 40.

6 L. Karim and N. Nasser: IET Commun. 6 (2012) 2149.

7 K. Maenaka: Sens. Mater. 28 (2016) 927.

8 A. S. Poornima and B. B. Amberker: IET Wireless Sens. Syst. 1 (2011) 85.

9 M. Zorzi, P. Casari, N. Baldo, and A. F. Harris: IEEE J. Selected Areas Commun. 26 (2008) 1754.

10 S. Taruna, K. Sheena, and G. N. Purohit: Comput. Sci. Eng. Appl. 67 (2012) 275.

11 Z. Liu and Z. D. Qiu: J. Commun. 29 (2008) 104.

12 R. K. Tripathi, Y. N. Singh, and N. K. Verma: Electron. Lett. 49 (2013) 299.

13 S. H. Javadi and A. Peiravi: IET Signal Process. 6 (2012) 743.

14 C. Conficoni, A. Bartolini, A. Tilli, C. Cavazzoni, and L. Benini: IEEE Trans. Ind. Inf. 4 (2016) 1299.

15 D. Kumar, T. C.Aseri, and R. B. Patel: Comput. Commun. 32 (2009) 662.

16 Z. Hong, Y. Li, and G. J. Zhang: Acta Automatica Sinica. 39 (2013) 454.

17 N. Yaakob, I. Khalil, and M. Atiquzzaman: IET Wireless Sens. Syst. 5 (2015) 124.

18 Y. Faheem and S. Boudjit: IET Wireless Sens. Syst. 5 (2015) 219. 